\title{
Precursors and outcomes of work engagement among nursing professionals-a cross-sectional study
}

\section{Terje Slåtten ( $\sim$ Terje.slatten@inn.no)}

Inland Norway University of Applied Sciences

\section{Gudbrand Lien}

Hogskolen i Innlandet - Studiested Lillehammer

\section{Research article}

Keywords: Work engagement, Turnover intention, Job satisfaction, Service quality, Collaborative climate, Organizational culture, Nursing professionals, Hospitals

Posted Date: September 4th, 2020

DOl: https://doi.org/10.21203/rs.3.rs-33425/v2

License: (c) (i) This work is licensed under a Creative Commons Attribution 4.0 International License.

Read Full License

Version of Record: A version of this preprint was published at BMC Health Services Research on January 4th, 2022. See the published version at https://doi.org/10.1186/s12913-021-07405-0. 


\section{Abstract}

Background: Health services organizations must understand how best to lower nursing professionals' turnover intentions, increase their job satisfaction and quality of care provided to patients. This study aims to examine whether work engagement, which refers to a positive fulfilling, work-related state of mind, is a significant predictor of the achievement of these preferred organizational goals. The study also aims to examine whether organizational culture and climate can manage the work engagement of nursing professionals and indirectly contribute to the accomplishment of the preferred organizational goals.

Methods: The proposed conceptual model was tested in a quantitative study in which nursing professionals participated. The data were analyzed using confirmatory factor analysis and structural equation modeling in Stata.

Results: The three key findings from this study were: i) work engagement of nursing professionals was found to be positively related to service quality of care $(b=0.551)$ and job satisfaction $(b=0.883)$. Job satisfaction fully mediates the relationship between work engagement and turnover intention and in itself explains almost $60 \%\left(R^{2}=0.59\right)$ of turnover intention (which refers to the intention to leave a job on a personal/workers level), ii) nursing professionals' perception of organizational culture $(b=0.278)$ and collaboration climate $(b=0.331)$ were both directly related to their work engagement, iii) work engagement fully mediates the relationship between organizational culture, organizational climate and service quality of care and job satisfaction. Moreover, work engagement particularly mediates the relationship between collaborative climate and job satisfaction.

Conclusions: The work engagement of nursing professionals is highly correlated, especially to their job satisfaction. Work engagement and intention to leave a job is (fully) mediated by job satisfaction. Employers should therefore focus on improving job satisfaction of nursing professionals. The work engagement of nursing professionals is a common key factor for improvements. Consequently, leaders and managers should continuously manage nursing professionals' work engagement, focusing on such areas as organizational culture and climate because work engagement is a substantial driver to enable multiple desirable outcomes for hospital organizations.

\section{Background}

Research has shown that a sustainable human resource base in healthcare organizations is a major challenge. Regarding this, Gaupta et al. noted: "Motivation, morale and retention ... have emerged as issues of significant concern for healthcare providers and policymakers" [1, p. 2807].

The difficulty in handling this major challenge of having a sustainable human resource base is especially relevant when considering the group of nursing professionals, which is characterized as a "critical part of the human resource base" [2, p. 2] in healthcare organizations. Several organizations and even countries reveal that healthcare organizations experience an increased level of turnover among nursing 
professionals [3] and characterize this challenge as an "ongoing problem" [3, p. 1180]. This fluctuation in nursing professionals in healthcare organizations has several negative impacts such as lowering the level of service quality provided to patients [4], lower patient satisfaction [5], reduction in productivity [6], negative effect on work morale and an increase in the level of intention to leave a job [7]. Consequently, it becomes essential for hospital organizations to identify those factors having the potential to contribute positively to achieving desired organizational goals such as lowering intention to leave the job among nursing professionals as well as being able to increase (or maintain) their job satisfaction and level of service quality provided to patients.

This paper seeks to examine whether the work engagement of nursing professionals has the potential to function as a core factor in the achievement of multiple desirable outcomes for hospital organizations. Work engagement is about the nursing professionals' perception of experiencing a positive response to the work they are performing. Work engagement has been proposed as an important factor for organizations to focus on. Kaye and Jordan-Evans noted the importance of work engagement by stating, "The challenge today is not just retaining talented people, but fully engaging them, capturing their minds and hearts at each stage of their work lives" [8]. Keyko et al. remarked the importance of work engagement of professional nursing in this way: "work engagement in professional nursing practice is critically important to consider when addressing key challenges of health systems" [9, p. 142]. Similarly, Bargagliotti stated, "work engagement is the central issue for $21^{\text {st }}$ century professionals and specifically for registered nurses" [10, p. 1414].

Previous research on work engagement among nursing professionals has linked work engagement to several aspects. In an extensive systematic review by Keyko et al. [9], the authors identified a total of 77 influencing factors and 17 outcomes associated with work engagement. In their paper, the authors developed a model to assist in pinpointing knowledge gaps in existing research to motivate and give direction to future research on work engagement. The extensive review by Keyko et al. [9] motivated the four specific aims of the present study.

First, Keyko et al. note a lack of 'research on patient-related outcomes of professional nurses' work engagement" [9, p. 161]. This study follows this explicit recommendation by examining service quality of care provided to patients as one type of outcome of nurses' work engagement. By doing this, it contributes as Keyko et al. also noted to "provide further support for the importance and value of promoting work engagement in professional nursing practice" [9, p. 161]. Second, based on their review, regarding research identifying antecedents and outcomes related to nurse engagement, Keyko et al. also noted that "North American studies currently dominate the research in this field." Consequently, they recommend performing more studies in other populations and settings. Based on these authors' suggestion, nurses' work engagement in this study is examined in a Norwegian hospital context and thus represents the Scandinavian population and setting. As such, it contributes to "analysis and discussion on potential geographical and cultural differences" [9, p. 161]. Third, although Keyko et al. identified 77 influencing factors to nurses' work engagement, the findings reveal that no study included in the review has examined how organizational culture is linked to nurses' work engagement. This is surprising 
because the "culture of an organization provides boundaries and guidelines that help members of the organization to know the correct way to behave towards each other, and how to perform their work tasks" [11, p. 2711]. According to Slåtten et al. [2, 12], healthcare organizations need to foster a culture and climate that focuses on frontline employees. Recently, internal market-oriented culture (IMOC) has been suggested as one type of culture that healthcare organizations should develop regarding their frontline employees [2,12]. Based on IMOC as one type of culture orientation, Slåtten et al. suggest that "future research could examine whether and how IMOC is connected to other concepts such as ... employees' individual and collective engagement" [12, p. 177]. This study follows this recommendation and accordingly contributes to revealing the linkage between IMOC and individual nurses' work engagement. Fourth, in their review, Keyko et al. also requested that future research on nurses' work engagement should include "more complex statistical analyses ... to improve the strength of study findings" [9, p. 161]. This study follows this recommendation by examining both precursors and outcomes of nurses' work engagement in the same research model and thus offers an opportunity to perform more complex and complete statistical tests of both direct and mediating effects related to nurses' work engagement.

Considering the abovementioned four aims, this paper contributes to extending and deepening previous research on nurses' work engagement and thus contributes both theoretically as well as suggesting practical implications to "enable development of initiatives that enhance work engagement and its outcomes within the current health care context" [9, p. 143].

This paper is structured in the following way. First, it starts by presenting the conceptual model of the study. Then, the concepts of nurses' work engagement are described and defined as well as other concepts proposed to be precursors and outcomes of work engagement. Then, the methodology and findings from our empirical study are presented. The paper ends with a discussion. This latter part also includes suggestions for future research as well as conclusions of the study.

\section{Conceptual Model}

Figure 1 visualizes the proposed conceptual model of this study. As seen at the foot of Figure 1, the elements in the conceptual model are grouped into three different categories, Appraisal-ResponseOutcome (ARO) that are connected in a specific directional manner as shown by the arrows between them. At the top of the figure, each of the three categories is labeled with names reflecting the content and focus in each category. The category Response (R) is named "individual-level resource" and is represented as the most central concept in this study, that is, work engagement (WE). Furthermore, the category Appraisal (A) is named as "organizational-level resources" and is represented by the concepts (i) internal market-oriented culture (IMOC) and (ii) collaboration climate (CC). It is notable to recognize that the term "resource" is used to label both organizational- and individual-level types of resources. The term "resource" signals something that is not static but dynamic and consequently varies, either positively or negatively, as time passes. Further, because a "resource" is dynamic, it also implies that a "resource" is potentially changeable or manageable. As seen in Figure 1, the appraisal of the two organizational-level resources (referring to IMOC and CC) is directly linked to the response in the individual-level resource 
(referring to nurses' WE). Three types of Outcomes (0) are linked to WE in this study. The category Outcomes ( 0 ) is named "organizational goals" and is represented by the three concepts (i) service quality of care (SQC), (ii) job satisfaction (JS) and (iii) turnover intention (TI). Notably, TI in this study does not refer to turnover related to an organization's intention to discharge/dismiss at the organizational level. Specifically, TI refers to professional nurses' intention to leave the job and thus refers to the personal/workers level. In the following, each concept and linkage in Figure 1 are elaborated in detail.

Work engagement (WE)

The concept of WE originally comes from positive psychology, which focuses on the positive resources and strengths of humans, rather than their limits [13]. Specifically, in this study, WE is defined as "positive fulfilling, work-related state of mind" [14, p. 74]. Moreover, this positive work-related state of mind embraces three interconnected types of feelings, namely, absorption, vigor and dedication. Absorption is about employees' level of interest and engrossment or immersion of employees in their work so that the sense of time is lost [15]. Vigor reflects the workers' level of energy and mental resilience while performing their work. Dedication describes how involved employees are in the sense of significance, inspiration and challenge [15]. It is important to note that work engagement is a "state of mind" [14, p. 74]. Defining the WE as a state of mind and not a trait of a person means that WE, compared with the relative stability of a trait of a person, is dynamic. This dynamic feature implies that all "ingredients" of the internal resource pool of individual employees' WE are potentially changeable and something that can fluctuate, both positively and negatively within a certain timeframe.

As indicated in the previous discussion and visualized in Figure 1, WE is a response (R) and an individuallevel resource. WE is heterogeneously distributed among individual employees in organizations. WE is a variable that can be described as a motivational-like variable with the potential to improve organizational outcomes positively [16]. In the next section, three outcomes of WE are proposed.

\section{The outcomes of work engagement (WE)}

WE is, as visualized in Figure 1, an individual-level resource that is suggested to be linked to three different types of outcomes, namely, (i) turnover intention (TI), (ii) job satisfaction (JS) and (iii) service quality of care (SQC). All outcomes of WE represent desirable organizational goals that healthcare organizations want to achieve.

Turnover intention (TI)

In this study, Tl embraces nurses' psychological responses to conditions in organizations [17] and consequently their reflection about whether they should stay with the organization that employs them. It is important to note that TI focuses on intention to leave a job on a personal/workers level. As such, TI reflects an important first cognitive step in the decision-making process [18] about leaving the organization. 
Previous research has suggested that WE is negatively related to TI. When employees experience a positive state of mind characterized by vigor, dedication and absorption, referring to those elements embraced by the concept WE, this should lead to a more favorable evaluation and thoughts about their workplace and thus be associated with decisions or intention to not leave the organization in which they are employed. Previous research on nurses supports the existence of a negative relationship between WE and turnover intention. For example, in their study, Moloney et al. [19], including 2876 nurses working in New Zealand as respondents, found that nurses' WE is negatively linked to the intention to leave the organization. Rodwell et al. [20] also found a negative linkage between nurses' WE and nurses' intention to leave the organization in their study of 459 nurses across Australia. Similar to the two aforementioned research studies, Wan et al. [6] found, when performing a study among 778 nurses employed in a hospital in China, a significant negative relationship between nurses' WE and TI. In line with the aforementioned research findings, it is expected to find a similar negative relationship between nurses' WE and TI among those nurses included in this study (referring to nurses from Norway). Therefore, the following hypothesis is proposed:

Hypothesis 1: WE (positive response to work) is significantly and negatively related to $\mathrm{TI}$ (intention to leave a job).

Job satisfaction (JS)

According to Tomietto et al. [16], job satisfaction (JS) requires "strategies ... to enhance job satisfaction ... one of the most challenging topics in nursing research" [16, p. 2]. In this study, WE is suggested to be one such strategy to achieve JS. Therefore, as seen in Figure 1, JS is proposed to be one outcome of nurses' WE. In this study, JS is about the "extent to which employees [nurses] like their jobs" [21, p. 13]. JS is a cognitive concept regarding nurses' job satisfaction. Specifically, JS refers to nurses' global assessment of their job [22].

Although JS has generally been included in several previous studies on nurses, it seems that relatively few of these have examined the specific link between WE and JS. For example, in their extensive review of antecedents and effects of work engagement by Keyko et al. [9], the authors only reported four previous studies examining this linkage among professional nurses. Of these four studies, two studies identified a significant relationship between WE and JS. One study found a nonsignificant relationship between WE and JS. One study was qualitative. Consequently, there is a need for more research on this topic that specifically focuses on the domain of nursing. In general, it seems that previous research most often has identified a positive relationship between WE and JS [23]. It is reasonable to assume a similar pattern and relationship between these concepts among nurses. Consequently, when positively experienced, the three "ingredients" of WE should lead employees to have more favorable thoughts and feelings about their job. It is difficult to imagine a situation where an employee has a high level of WE and at the same time, dislikes/hates his/her job and thus has low JS. In line with previous research [23] on this topic, this study assumes that professional nurses' WE is positively related to their JS. This reasoning leads to the second hypothesis in this study: 
Hypothesis 2a:WE (positive response to work) is significantly and positively related to job satisfaction (JS).

Previous research has found that the JS of nurses is directly linked to TI among nurses [16]. However, in this study, it is also suggested that JS functions as a mediator between WE and TI. This represents a complementary "route" to TI compared with what was proposed in Hypothesis one. In addition, to have a direct impact on $\mathrm{Tl}$, it is also assumed that there is some inner mechanism in humans that explains why. there exists a relationship between WE and TI. In this study, this inner mechanism in humans refers to JS. JS does not arise by itself or occur in isolation. JS is always triggered or created by someone or something. In this study, "something" refers to nurses' WE. As discussed in the second hypothesis, previous research on WE suggested its impact on JS [9, 23]. Based on this, when nurses experience a positive WE, their JS will be positively affected. Further, when JS increases because of an increase in WE, it is reasonable to assume that this will also lower the level of TI among nurses. In the study by Tomietto et al. [16], the authors conceptualize JS as one potential mediator between nurses' WE and nurses' TI. Nevertheless, the authors did not make any empirical test of whether JS was operating as a mediator. Consequently, this study is the first study to test whether JS is operating as a mediator between WE and TI. The aforementioned reasoning can be expressed by this formal hypothesis:

Hypothesis $\mathbf{2 b}$ :The relationship between work engagement (WE) and turnover intention (TI) is mediated by job satisfaction (JS).

Service quality of care (SQC)

The best level of SQC to patients is a highly desirable organizational goal for hospital organizations to achieve. Nursing professionals make a considerable contribution to the total "service package" regarding SQC. According to Chen et al., this group of frontline workers "tend to have the longest and closest contact with patients" $[24$, p. 1]. In this study, the concept of SQC refers to nurses' perceptions of the overall quality of services provided. Although this is a subjective in contrast to an objective way to capture the content of SQC, it follows from how previous research sometimes has studied service quality both within and outside healthcare and other contexts [2, 25]. Moreover, previous research also suggests that there exists a "psychosocial closeness" between providers' and receivers' perception of service quality [26]. Consequently, it is assumed in this study that nursing professionals are capable of considering whether their level of quality of services is of high or low standard or lies within a zone that "customers generally perceive as acceptable" [27, p. 208]. The assumption about the association is formulated by this hypothesis:

Hypothesis 3:Work engagement (WE) is positively related to service quality of care (SQC).

\section{Precursors to work engagement (WE)}

In Figure 1, precursors to WE are suggested to be (1) collaboration climate (CC) and (2) internal marketoriented culture (IMOC). Consequently, the discussion is focused on whether the appraisal of CC and 
IMOC, representing two organizational-level resources, promotes or causes a response in the individuallevel resources of employees referred to as WE in Figure 1. This is discussed in the following.

Collaboration climate (CC)

An organizational climate is about employees' shared perceptions of their organization. This shared perception of climate varies from strongly negative to strongly positive. The literature emphasizes the significance and value for organizations to focus on organizational climate. For example, in the research undertaken by Kieft et al., the authors found that "it is important to develop and maintain collaborative working relationships with professionals, including those in their own field" [28, p. 5]. Consequently, there are good reasons to include collaboration climate (CC) and how frontline employees perceive and appraise this organizational-level resource. However, in an organization, there are a variety of alternative aspects to focus on when studying a collaborative climate. Therefore, it is necessary to identify aspects that are both relevant and specific for the phenomenon in focus. Consequently, "climate is best regarded as a specific construct having a referent" [29, p. 1278]. The literature suggests several ways to study organizational climate. For example, D'Amour et al. suggest four ways to analyze cooperation in healthcare organizations [30]. In this study, CC is about two work-related concerns regarding interdepartmental collaboration in the health organization. Specifically, CC refers to frontline employees' perception of interdepartmental (i) conflict and (ii) connectedness, both of which are suggested in previous research [31,32]. The first climatic aspect of "conflict" of CC is about whether there exist tensions, caused by inconsistency regarding actual and desired responses between departments in the organization. The second climatic aspect, "connectedness," focuses on whether there are formal and informal contacts across departments in the organization. This latter aspect intends to reveal nursing professionals' perceptions of whether departments are operating dependently or independently from each other. The two aspects of CC represent and reflect nursing professionals' perceptions of the supportive work environment or what alternatively can also be labeled as the internal service climate in an organization [29].

According to the job demands-resources (JD-R) model, WE is fostered by job resources [33]. In this study, CC represents this organizational level of resource in the JD-R model that promotes or fosters WE among frontline employees. According to Wan et al., "a supportive work environment ... offers various resources to foster employees' willingness to dedicate their efforts and abilities to job tasks" [6, p. 1334]. Although it seems that no study has examined the link between the (work environmental factor) CC and WE specifically, previous research has found that the work environment, in general, is positively associated with WE. For example, the work environment has been found to be associated with WE among nurses [34]. Furthermore, research has found that perceptions of organizational climate are linked to employees' attitudes [35]. Consequently, based on the JD-R model and findings in previous research there are good reasons to expect CC to be positively associated with WE. Therefore, the following hypothesis is proposed:

Hypothesis 4:Collaboration climate (CC) is positively related to work engagement (WE). 
Internal market-oriented culture (IMOC)

In Figure 1, the internal market-oriented culture (IMOC) is considered as an organizational-level resource. Organizational culture is, according to Banaszak-Holl, said to "pervade all aspects of organizational life" [36, p. 462]. Organizational culture is a "stable element deeply rooted in employees' mentality" [37, p. 585]. Moreover, organizational culture embraces norms that "provide the rules for behavior" [38, p. 2]. Of the different components an organizational culture consists of, norms and behavior are the two most observable components [39]. In this study, organizational culture refers to nursing professionals' perception of norm-based behavior regarding the IMOC in the organization. As such, as also noted in Slåtten et al., IMOC "focus on more tangible or visible aspects of organizational culture that frontline employees of hospitals experience or observe daily" [12, p. 160]. The basic idea with the concept of IMOC is to treat employees in organizations as customers. Parallel to external customers, it is important to treat these internal customers (referring to employees) in the best possible way. Consequently, IMOC focuses on employees' perception of whether managers' norm-based behavior in the organization is oriented toward satisfying the needs and wants relevant to employees' working conditions [12]. Three systems constitute the norm-based behavior concept of IMOC: (i) internal-market intelligence generation, (ii) internal-intelligence dissemination and (iii) response to internal intelligence [40]. The three systems are closely connected and represent a logical flow of information from system one to system three. The first system is about management-related activities to collect information regarding the needs and wants of employees. The second system is about management interpretation and understanding of employees' needs and wants. Finally, the third system is about the willingness and capability of the management in an organization to take steps to perform real actions and actively do what is necessary to satisfy the needs and wants of their employees. All systems of IMOC are interconnected. However, system three (referring to "response to internal intelligence") is probably the most visible part employees experience or observe in their day-to-day work and thus most prominently brings IMOC to "life" in the organization.

Organizational culture "strongly influences employee behaviors" [39, p. 1] and thus is an employeeimpacting instrument to create the desirable and necessary behavior in an organization. Previous research within healthcare organizations has found organization culture associated with such areas as job satisfaction, leadership behavior, turnover intention and organizational attractiveness ([2], [12], [38]). Regarding this and specifically referring to IMOC, Slåtten et al. state, "IMOC is a type of organizational culture affecting frontline employees" [12, p. 161]. The core of IMOC is about management's ability to satisfy needs and wants with a specific focus on employees' work roles. With this in mind, it is reasonable to assume that when IMOC is perceived by employees as something good, it should positively influence employees' vigor, absorption and dedication, which all are embraced in the concept of employees' WE. The association between IMOC and WE is also supported in the JD-R model. The JD-R model [33] highlights that different resources in the work environment can promote or act as motivational factors for nursing professionals' dedication and efforts (or what this study refers to as WE) to perform work tasks [33]. In this study, IMOC is termed an organizational-level resource. Therefore, based on the JD-R model and previous research, IMOC should have an impact on WE. This reasoning leads to the following hypothesis: 
Hypothesis 5a:Internal market-oriented culture (IMOC) is positively related to work engagement (WE).

Although IMOC is expected to be directly associated with WE, it is also assumed that this relationship is mediated through the concept of organizational climate, in this study represented as CC. Accordingly, it is proposed that two "routes" exist from IMOC to WE. In the literature, the concept of climate and culture are often suggested to be closely related. Regarding conceptual closeness Carlucci noted, "culture and climate are similar concepts" [37, p. 585]. Although they at first sight seem to be rather similar, they diverge. Culture is about relatively stable and deeply rooted norm-based behavior while climate is more "superficial elements such as employees' reactions, opinions and tendencies" [37, p. 585]. Therefore, the climate is a surface manifestation of culture [37]. Based on this, it is expected that, when employees perceive IMOC in a positive manner, this would have a positive impact on the organizational climate of CC. Moreover, when CC increases, because of IMOC, this should have a positive impact on employees' WE. To the authors' knowledge, no previous research has examined this specific linkage. However, two arguments support this idea. First, IMOC as defined in this study focuses on managers' norm-based behavior and their orientation toward satisfying the needs and wants in employees' work roles. Consequently, the norm-based behavior of management, manifested in IMOC, models the "correct standard" of organizational climate for all employees such as how CC should be in the organization. As such, and based on social learning theory [41], employees learn appropriate behavior from their managers as significant role models. When IMOC and CC are perceived as positive, this should significantly increase employees' WE. The second argument supporting a linkage between the three variables can be found in the JD-R model. IMOC and CC are both based on the JD-R model, suggested to be resources. In Figure 1 , they are both labeled as organizational-level resources. However, as indicated in the aforementioned discussion, IMOC is critical for CC in the organization. Thus, the resources of IMOC serve as necessary input or "ingredients" to positively build and increase the CC resources. Consequently, when the (resources in) CC increase because of a positive impact of (the resources in) IMOC, this should lead to a positive increase in employees' absorption, vigor and dedication which are all embraced in the concept of WE. Thus, IMOC and CC are expected to work in tandem to strengthen employees' WE. The assumption about WE as a mediator between IMOC and CC can be formulated by this formal hypothesis:

Hypothesis 5b:The relationship between internal market-oriented culture (IMOC) and work engagement (WE) is mediated by collaboration climate (CC).

WE as a mediator between IMOC, CC and SQC, TI and JS

As visualized in Figure 1, WE is suggested to play a role as a mediator between the two organizationallevel resources (IMOC and CC) and the three organizational goals (SQC, TI and JS). WE, labeled as an individual-level resource, is expected to play a central key role. Consequently, WE is assumed to function as the mediating mechanism that binds or ties the suggested variables together to a whole.

As noted in the previous discussion, IMOC and CC are closely related concepts. Because of their close relationship and association, it is reasonable to assume that the two concepts should work in the same direction depending on how employees appraise or perceive them. As Trus et al. noted, "culture and 
climate represent a social context ... that constrains and promotes certain behaviors and interactions" [42, p. 55]. This study takes a positive perspective when studying the social context of IMOC and CC. Consequently, rooted in the assumption of the close relationship between IMOC and CC, it is expected that both will be found as positive promoters of employees' WE. Moreover, when WE increases, because of employees' positive perception or appraisals of IMOC and CC, this should also lead to several positive job-related outcomes and thus an achievement of organizational goals such as increased SQC, JS and reduced TI of employees in the organization.

The assumption of the mediating role of WE is summarized in these two final hypotheses:

Hypothesis 6: Work engagement (WE) functions as a mediator between internal market-oriented culture (IMOC) and a) service quality of care (SQC), b) turnover intention (TI) and job satisfaction (JS).

Hypothesis 7:Work engagement (WE) functions as a mediator between collaboration climate (CC) and a) service quality of care (SQC), b) turnover intention (TI) and job satisfaction (JS).

\section{Methods}

\section{Design and setting of the study}

The data for this study were collected from four public hospitals located in southeast Norway.

The process to design and develop the survey for this study included several workshops. Participants from both academia and the target group were represented in the workshops. Combining capabilities from these two groups contributed to developing and designing a more appropriate tailor-made survey. Most questions/instruments used were based on previous research. However, the questions/instruments had not been previously used in a healthcare context. As a consequence of this, through the research process to make sure to develop a tailor-made questionnaire, several changes had to be made in the best possible way to adapt and modify instruments to the selected population of participants. The final questionnaire was pretested on part of the target group. The pretest resulted in some minor changes. No questionnaire used in the pretest was included in the final analysis or test of the hypotheses.

The final questionnaire was distributed by e-mail to the nursing staff. All invited participants were informed about the background, aim and focus of the study. Furthermore, the shared information clearly indicated that all participation was voluntary and all information collected in this research project would be handled strictly confidentially. To collect the data, the software Checkbox was used. One hundred sixty-four questionnaires were received. All returned questionnaires had a satisfactory quality and, consequently, all were included as the basis for testing the proposed hypotheses.

\section{Instruments}

In this study, JS refers to nursing professionals' global assessment of their job. Items for JS are based on and adapted from Anaza and Rutherford [22]. The concept of IMOC refers to nursing professionals' 
perception of managers' norm-based behavior regarding the IMOC reflected in three aspects: (i) internalmarket intelligence generation, (ii) internal-intelligence dissemination and (iii) response to internal intelligence. The items included in IMOC are based on and adapted from Gounaris [40]. The concept of WE in this study was defined as "positive fulfilling, work-related state of mind" [14, p. 74] and represented three facets of state of mind: (i) absorption, (ii) vigor and (iii) dedication. Items included in WE are based on Schaufeli et al. [14] and modified for this study. In this study, the concept of SQC is about nursing professionals' perceptions of their overall service quality provided. Items included in SQC are based on Slåtten [43]. The concept of CC refers to nursing professionals' perception of two aspects of interdepartmental collaboration: (i) level of conflict and (ii) level of connectedness. These two aspects are suggested in previous research. Items for CC are based on the work of Kohli and Jaworski [31, 32] and modified for this study. The concept of TI reflects frontline employees' cognitive reflection about whether they should stay with the organization that employs them. Items included in $\mathrm{TI}$ are based on Boshoff and Allen [44]. All items for each construct were measured using a Likert scale from (1) strongly disagree to (7) strongly agree. Table 2 lists all items included in each construct.

\section{Data analysis}

Structural equation modeling was applied to explore the relationships among the constructs. The first step was to assess the measurement model (consisting of reflective latent constructs); step two tested the structural model. The estimation was done with the "sem" package in Stata [45]. Testing of the mediation hypotheses was conducted using the "medsem" package in Stata [46], following the approach proposed by Zhao et al. [47].

The measurement model was assessed by examining several criteria. Goodness-of-fit indices: SRMR, RMSEA, CFI, TFI; indicator reliability (item loading); latent construct reliability (Raykov's reliability coefficient); convergent validity (average variance extracted (AVE, all AVE values should be larger than the squared correlations among the latent constructs)); and discriminant validity. Convergent and discriminant validity make up the construct validity. The "rules of thumb" used in Tables 2 and 3 are based on Mehmetoglu and Jacobsen [45].

The structural model was assessed with the same goodness-of-fit measures as the measurement model. The structural model's path coefficients reported are standardized values, which ranged between -1 and 1. The closer a path coefficient is to \pm 1 , the stronger is the relationship.

\section{Results}

\section{Characteristics of the study sample}

Table 1 shows the personal characteristics of the study sample. Most responses were received from those employees who worked as nurses or specialist nurses. A large portion of nursing professionals had significant experience. Of the total sample, $64 \%$ had worked in the investigated hospitals for more than 
10 years. About $50 \%$ worked full-time. Some $35 \%$ were under 40 years of age, about $30 \%$ were between 41 and 50 , and about $35 \%$ were older than 50 .

Table 1 Personal characteristics of the study sample $(N=164)$

\begin{tabular}{llr}
\hline & & $\%$ \\
\hline Sex: & Female & 93.3 \\
& Male & 6.7 \\
Work as: & Nurse & 43.9 \\
& Specialist nurse & 49.4 \\
\hline Employed: & Midwife & 6.7 \\
\hline & less than 5 years & 20.7 \\
& between 6 and 10 years & 15.3 \\
\hline Part-time or full-time: & more than 10 years & 64.0 \\
\cline { 2 - 3 } & part-time job & 50.6 \\
Age: & younger than 40 years & 49.4 \\
\hline & between 41 and 50 years & 29.9 \\
& older than 50 years & 35.3 \\
\hline
\end{tabular}

\section{Measurement model}

Table 2 shows that the reliability and validity measures were all within the rules of thumb, and Table 3 shows that the goodness-of-fit indices were also within the commonly accepted thresholds, indicating that a sound measurement model was established.

Table 2 Results of the measurement model for the constructs internal market-oriented culture, collaboration climate, work engagement, service quality of care, turnover intention and job satisfaction 


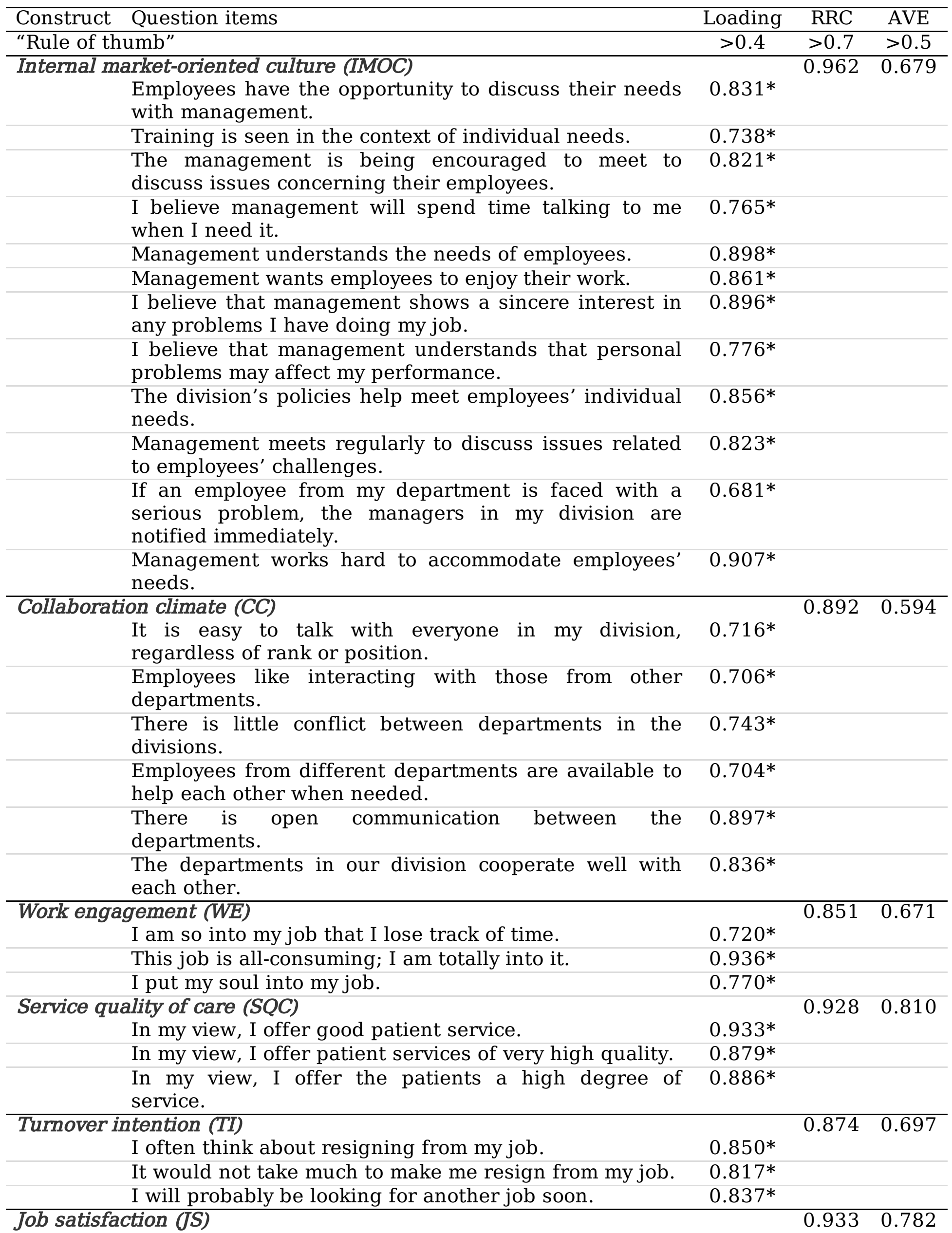




\begin{tabular}{|c|c|c|c|}
\hline Construct Question items & Loading & RRC & AVE \\
\hline "Rule of thumb" & $>0.4$ & $>0.7$ & $>0.5$ \\
\hline $\begin{array}{l}\text { If a good friend of mine were interested in a job like } \\
\text { mine in this organization, I would strongly recommend } \\
\text { it. }\end{array}$ & $0.865^{*}$ & & \\
\hline My job is the sort of job I wanted when I took it. & $0.896 *$ & & \\
\hline $\begin{array}{l}\text { If I had to decide all over again whether to take a job in } \\
\text { this organization, I would. }\end{array}$ & $0.868 *$ & & \\
\hline Overall, I am satisfied with my current job. & $0.908^{*}$ & & \\
\hline
\end{tabular}

$* p<0.05 . \mathrm{RRC}=$ Raykov's reliability coefficient. AVE $=$ Average variance extracted.

Table 3 Summary of the fit statistics of the measurement and structural models

\begin{tabular}{|c|c|c|c|c|c|}
\hline Model & d.f. & RMSEA & CFI & TLI & SRMR \\
\hline Fit criteria ("Rule of thumb") & & $<0.1$ & $>0.9$ & $>0.9$ & $<0.1$ \\
\hline Measurement model 683.85 & 419 & 0.062 & 0.941 & 0.934 & 0.055 \\
\hline Structural model & 427 & 0.065 & 0.934 & 0.928 & 0.085 \\
\hline
\end{tabular}

d.f., degrees of freedom; RMSEA, root mean square error of approximation; CFI, comparative fit index; TLI, Tucker-Lewis index; SRMR, standardized root mean square residual.

\section{Structural model}

Table 3 indicates that the goodness-of-fit indices for the structural model were also within the commonly accepted thresholds. Figure 2 presents the standardized path coefficients and their significance level. We found that IMOC and CC had a positive and statistically significant and about equal effect on WE, and thus support hypotheses $\mathrm{H}_{5 a}$ and $\mathrm{H}_{4}$. WE had a large and statistically significant effect on both SQC and JS, and thus supports $\mathrm{H}_{3}$ and $\mathrm{H}_{2 a}$. The relationship between WE and TI was not significant and $\mathrm{H}_{1}$ was not supported. The model explains $32 \%$ of the variance in WE, $30 \%$ of the variance in SOC, $59 \%$ of the variance in $\mathrm{TI}$ and $69 \%$ of the variance in JS.

Using mediation analysis, Table 4 showed that the indirect effect of job satisfaction on the relationship between work engagement and turnover intention was significant $(b=-0.501)$, implying a full mediator effect and support for $\mathrm{H}_{2 b}$. Collaboration climate showed a significant indirect effect $(b=0.207)$ and $a$ partial mediation effect between internal market-oriented culture and work engagement, and thus $\mathrm{H}_{5 \mathrm{~b}}$ received support.

We tested a total of six mediator effects of work engagement, and our findings (Table 4) show in general that work engagement is a quite strong mediator. Work engagement had a significant indirect $(b=0.207)$ and a full mediation effect on the relationship between internal market-oriented culture and service quality of care, supporting $\mathrm{H}_{6 \mathrm{a}}$. Work engagement does not mediate the relationship between internal market-oriented culture and turnover intention, and then no support of $\mathrm{H}_{6 \mathrm{~b}}$. However, work engagement intervenes between internal market-oriented culture and job satisfaction (with an indirect effect of $b=$ 0.178), supporting $\mathrm{H}_{6 \mathrm{c}}$. Work engagement fully mediates between collaboration climate and service 
quality of care (with an indirect effect of $b=0.145$ ), supporting $\mathrm{H}_{7 \mathrm{a}}$. The relationship between collaboration climate and turnover intention had no significant indirect effect and was then not mediated by work engagement and we got no support for $\mathrm{H}_{7 \mathrm{~b}}$. Lastly, work engagement partially mediates the relationship between collaboration climate and job satisfaction, with an indirect effect of $b=0.183$, supporting $\mathrm{H}_{7 \mathrm{~b}}$.

Table 4 Mediator analysis. Standardized direct, indirect and total effects

\begin{tabular}{|c|c|c|c|c|c|c|}
\hline Hypothesis & Effect $^{\mathrm{a}}$ & Mediator & $\begin{array}{l}\text { Direct } \\
\text { effect }^{\mathrm{a}}\end{array}$ & $\begin{array}{l}\text { Indirect } \\
\text { effect }\end{array}$ & $\begin{array}{l}\text { Total } \\
\text { effect }\end{array}$ & $\begin{array}{l}\text { Mediator } \\
\text { effect }^{b}\end{array}$ \\
\hline $\mathrm{H}_{2 b}$ & $\overline{\mathrm{WE}}{ }^{\circledR} \mathrm{TI}$ & JS & 0.085 & $-0.501^{* * *}$ & 0.416 & Full \\
\hline $\mathrm{H}_{5 b}$ & IMOC $® \mathrm{WE}$ & $\mathrm{CC}$ & $0.275^{* *}$ & $0.207 * *$ & 0.482 & Partial \\
\hline $\mathrm{H}_{6 \mathrm{a}}$ & IMOC ${ }^{\circledR}$ SQC & WE & -0.155 & $0.141 * *$ & 0.014 & Full \\
\hline $\mathrm{H}_{6 \mathrm{~b}}$ & IMOC $® \mathrm{TI}$ & WE & -0.179 & 0.023 & -0.156 & No \\
\hline $\mathrm{H}_{6 \mathrm{c}}$ & IMOC $®$ JS & $\mathrm{WE}$ & 0.111 & $0.178^{* *}$ & 0.289 & Full \\
\hline $\mathrm{H}_{7 \mathrm{a}}$ & $\mathrm{CC} ® \mathrm{SQC}$ & WE & 0.211 & $0.145^{* *}$ & 0.356 & Full \\
\hline $\mathrm{H}_{7 \mathrm{~b}}$ & $\mathrm{CC} \AA \mathrm{TI}$ & WE & 0.069 & 0.024 & 0.093 & No \\
\hline $\mathrm{H}_{7 \mathrm{c}}$ & $\mathrm{CC} \AA \mathrm{JS}$ & WE & $0.223^{* * *}$ & $0.183^{* *}$ & 0.406 & Partial \\
\hline
\end{tabular}

Notes: $\mathrm{IMOC}=$ Internal market-oriented culture; $\mathrm{CC}=$ Collaboration climate; $\mathrm{WE}=$ Work engagement; $\mathrm{SQC}=$ Service quality of care; TI = Turnover intention; JS = Job satisfaction.

** $p<0.05,{ }^{* * *} p<0.01$ are significance levels.

a The direct effects (the links $X ® Y$ ) in our basic structural model (Figure 2) are almost identical in our model for mediation analysis.

$\mathrm{b}$ We used the bootstrapping test of Zhao et al. [47] to test mediation. Briefly, this approach tests, through bootstrapping, if the direct and indirect effects are statistically significant, and the combination of these two tests decides if there exists no, partial or full mediation.

\section{Discussion}

To the authors' knowledge, this is the first study that undertakes a comprehensive test that includes both several direct and indirect effects associated with WE of nursing professionals in the same study. By doing so, it contributes to both extending and deepening research on this topic in healthcare services research. Specifically, it responds directly to Keyko et al.'s call for further research focused on "the importance ... of ... work engagement" [9, p. 161].

The contributions in this study can be divided into three sub-contributions. First, it embraces several different types of organizational outcomes associated with nursing professionals' WE. Specifically, it includes both patient-related outcomes (represented by SQC), job-related outcomes (represented by JS) and employer-related outcomes (represented by $\mathrm{TI}$ ). Doing so provides new insight into how nursing professionals' WE is associated with a variety of desired goals in healthcare organizations. Second, it contributes to revealing how nursing professionals' perception of organizational culture (represented by IMOC) and organizational climate (represented by $C C$ ) is acting as a precursor to their WE. Thus, it enhances our understanding of how potential strategies of the individual-level resource of WE can be cultivated and managed by the two organizational-level resources (referring to IMOC and CC). Third, in 
line with the overall aim and focus, the study has undertaken an extensive test of the centrality or role of healthcare professionals' work engagement in healthcare organizations. Specifically, using complex statistical tests and analysis, it contributes to answering whether WE functions as a full or partial mediator between organizational-level resources (IMOC and CC) and the three organizational goals (SQC, $\mathrm{TI}$ and JS) included in the study. As such, the study provides a comprehensive contribution regarding the true "value of $1 / 4$ work engagement" [9, p. 161] for healthcare organizations.

In line with much of the previous research, the individual-level resource of nursing professionals' WE is defined as "positive fulfilling, work-related state of mind" [14, p. 74]. Previous research has suggested that this positive state of mind embraced in WE is generally able to "improve ... organizational outcomes" [16, p. 2]. However, when considering the domain of healthcare service research, there has been a specific lack of studies on the outcomes of WE of nursing professionals and "customer" or patient-related outcomes. Regarding this, in their review of WE, Keyko et al. [9] commented: "one surprising finding of this review was the lack of research on patient-related outcomes." In contrast to the lack of this focus in previous research, this work contributes by including SQC as a patient-related outcome. The findings provide support for a linkage between WE and SQC $(b=0.551)$. WE explains $30 \%$ of the variance in SQC. As such, it provides empirical support for the positive impact of WE. Specifically, it contributes to revealing the value of having an engaged workforce and how healthcare organizations can capitalize on having an engaged workforce because it is capable of substantially increasing patients' experience and perception of service quality provided.

As this study also reveals, the value of WE of nursing professionals is not restricted only to having an impact on the patients' perception of service quality of the healthcare organizations. The results show that WE among nursing professionals also has a positive impact on their internal values, referring to their job satisfaction. JS was in this study defined as to what "extent employees like their jobs" [21, p. 13]. WE was found to be strongly linked to JS of healthcare professionals $(b=0.833)$. WE explains almost $70 \%$ $\left(R^{2}=0.69\right)$ of the variance in nursing professionals' JS, which can be characterized as substantial. Consequently, this implies that when leaders in health organizations know the level of WE of their nursing professionals, it is simultaneously telling something about whether they are satisfied with their job. Thus, WE is a predictor and can function like an organizational "thermometer" that leaders of healthcare organizations can actively use to identify (negative or positive) symptoms and trends regarding how healthcare professionals experience their work-related conditions in their organizations.

The results from this study show that JS is also linked to TI of nursing professionals $(b=-0.843)$ explaining almost $60 \%\left(R^{2}=0.59\right)$ of the variance in TI. Specifically, JS was found to act as a mediator between WE and TI. No significant linkage was identified between the direct effect of WE on TI. Most often, WE is linked individually to both $\operatorname{TI}[6,20,19]$ and JS $[9,23]$. Consequently, this lack of significant findings differs from previous research. However, in contrast to most previous research, this study undertakes a considerably more complex and comprehensive test of how WE is potentially associated with $\mathrm{TI}$, suggesting JS as a mediator. Previous research on this is limited because it has only theoretically conceptualized JS as a mediator between WE and TI (e.g., [16]). To the authors' knowledge, no study in 
health services research has empirically examined whether JS of nursing professionals mediates the relationship between WE and TI. Consequently, this study contributes to revealing an alternative and a more nuanced understanding and a more complex explanation, of how WE is linked to TI. Although WE and JS are conceptually different, they share a commonality because both focus on nursing professionals' perception of aspects of their work role. In contrast, $\mathrm{TI}$ is about the nursing professionals' perception of their employer and organization and whether they evaluate this as a great place to work. Consequently, $\mathrm{TI}$ is related to a "decision $1 / 4$ process" [18, p. 23]. Based on these distinctions, it is reasonable to assume that JS is closer to and more significantly an integral part of nursing professionals' decision process embraced in TI compared with WE. Therefore, it is both natural and more logical, as this study also has revealed, that JS is functioning as a mediator between WE and TI. Accordingly, JS of nursing professionals, stemming from the level of WE, is linked to individuals' decision processes about whether they should either continue or stop working for an organization they are employed in at present.

As noted previously in this paper, WE as an individual-level resource is considered and described as something that is not static but dynamic. This dynamic aspect implies that a WE of nursing professionals is potentially changeable or manageable. This study contributes to empirically demonstrating that this assumption seems to be correct. Both IMOC and CC, representing two types of organizational-level resources can positively change or "manage" WE of nursing professionals. The findings also contribute to revealing a close association between organizational culture (refer to IMOC) and organizational climate (refer to $\mathrm{CC}$ ). Simultaneously, it also reveals how organizational culture (IMOC) has an impact on WE through organizational climate (CC). IMOC and CC explain more than $30 \%$ $\left(R^{2}=0.32\right)$ of the variance in WE. Although the concept of organizational culture and organizational climate, in general, has been linked to WE in previous research $[6,12,34]$, very little research has been undertaken within the domain of healthcare. To the authors' knowledge, this is the first study in healthcare service research that includes and examines both IMOC and CC in the same study. By doing this, it contributes to our understanding and knowledge, revealing a multifaceted pattern of how IMOC and CC are linked to WE of nursing professionals. As such, the study directly contributes to Slåtten et al.'s $[12$, p. 177] call for more research on IMOC and WE. Further, it also contributes to increasing the amount of research on organizational culture studies in healthcare settings. Mesfin et al. recently noted that "there are only a few studies [on organizational culture] in a healthcare setting" [48, p. 3]. In addition to these explicitly demanded calls for more research, it also extends previous research on WE in another manner. Specifically, it contributes to revealing the extent of centrality or strength of WE when considering two organizational-level resources, IMOC and CC, and the actual achievement of organizational goals SQC, TI and JS. The findings reveal that the WE of nursing professionals, with one exception (refer to TI), mediates all the suggested relationships in the proposed model. This finding is a significant contribution both to research on WE in health service research and specifically to identifying the role that WE of nursing professionals seems to play for the achievement of desirable organizational goals in health organizations. Consequently, leaders and managers of healthcare service organizations should continuously have WE of their nursing professionals on their meeting agenda because it seems to be a core variable and strategic key tool to enable multiple desirable organizational outcomes. In summary, 
this study has important implications of how to handle one of the biggest challenges today's organizations are facing, mentioned in Kaye and Jordan-Evans, stating, "The challenge today is not just retaining talented people, but fully engaging them, capturing their minds and hearts at each stage of their work lives" [8].

\section{Limitations And Future Research}

Although this study contributes to health service research by including several interesting and important precursors and outcomes associated with WE, there is clearly more research that should be undertaken on the WE topic in future research.

First, this study limited its focus on WE of health professionals from an individual-level perspective. While this has been the most conventional way to study WE, other levels could be included. One such approach would be to focus on the WE from an organizational-level perspective and what the literature refers to as collective work engagement. This implies a shift in focus from "I" or individual engagement to "We" and an organizational climate engagement. In general, there is limited research undertaken focusing on collective engagement [49]. To the authors' knowledge, no study has been undertaken within health service research that specifically has focused on aspects associated with health professionals' collective engagement. Research outside health service research, but within knowledge-based firms, has revealed that collective engagement is positively associated with such areas as relationship learning, firms' innovative capability and employee commitment [50]. Given these positive outcomes, it is strongly recommended that future research in health services also includes a focus on collective engagement. One concrete suggestion of study would be, as also suggested in Slåtten et al. [12], to "examine whether and how IMOC is connected to ... collective engagement" [12, p. 177]. Such a focus, among several other potential factors, would clearly contribute to broadening our understanding of the concept of WE.

Second, according to Mesfin et al., "a good hospital culture leads to better individual performance" [48, p. 3]. This study limits its focus to study organizational culture represented by the concept of IMOC. Future research could include other potential types of organizational culture. One concrete suggestion would be to include one or more of the four organizational culture typologies (that is, 1) Clan, 2) Adhocracy, 3) Market and 4) Hierarchy) mentioned in Cameron and Quinn, known as "the competing value framework" [51]. Studying these organizational culture typologies and their association with potential antecedents or outcome variables contributes to extending our understanding about what type of culture should be put in place to develop and manage employees' WE for the achievement of organizational goals in healthcare organizations.

Third, this study only includes the concepts of culture and climate as drivers of WE. Consequently, examining the impact of leadership is left out and therefore represents a limitation of the study. Although one could argue that both IMOC and CC are affected by leadership, future research should clearly include leadership as a precursor to WE. The literature provides a range of potential leadership perspectives and styles that could be recommended to be included such as transformational leadership, ambidextrous 
leadership, transactional leadership, empowering leadership and leadership autonomy support. However, one concrete suggestion would be to include the four leadership styles mentioned in the Path-Goal Theory Conceptual Framework (that is, 1) Directive, 2) Achievement-oriented, 3) Participative and 4) Supportive) [52]. Similar to the suggestion including other types of organizational culture, examining a range of leadership styles would extend our insight of "what to do" in the best possible way to manage health professionals' WE for the achievement of organizational goals.

\section{Conclusions}

This study contributes to examining whether work engagement of nursing professionals is a core factor in the achievement of the preferred organizational goals in healthcare organizations. Work engagement was found to be correlated both to nursing professionals' level of service quality and their job satisfaction. Work engagement and turnover intention (referring to intention to leave a job) are mediated by job satisfaction. Based on the findings, leaders and managers of hospital organizations should manage nursing professionals' work engagement, focusing on organizational culture and climate. In conclusion, work engagement of nursing professionals can be characterized as a key factor because it constitutes a significant promotor to enable multiple desirable outcomes for hospital organizations.

\section{Declarations}

\section{Ethics and consent to participate}

This study was approved by the Norwegian Social Science Data Services (NSD). The NSD is a resource center and ethics committee for academic research in Norway. The NSD project number is 42091 . In accordance with The Personal Data Act $\S \S 2-7$ and 8 no. 1, the participants were given written information about the project and gave their consent by choosing to participate actively in the study by answering the online questionnaire.

\section{Consent for publication}

Not applicable

\section{Competing interests}

The authors declare that they have no competing interests.

\section{Funding}

Not applicable

\section{Availability of data and material}


The datasets used and/or analyzed during the current study are available from the corresponding author on reasonable request.

\section{Authors' contributions}

TS led the development and mainly drafted this manuscript. GL did the statistical analysis and contributed to interpreting the data. Both authors approved the final draft.

\section{Acknowledgments}

The authors express their gratitude to the study subjects for their participation in this research.

\section{Authors' information}

${ }^{1}$ Inland School of Business and Social Science, Inland Norway University of Applied Sciences, Lillehammer, Norway

\section{Abbreviations}

IMOC: Internal market-oriented culture; CC: Collaboration climate; WE: Work engagement; SQC: Service quality of care; TI: Turnover intention; JS: Job satisfaction; SEM: Structural equation modeling; SRMR: Standardized root mean square residual; RMSEA: Root mean square error of approximation; CFI: Comparative fit index; TLI: Tucker-Lewis index; RCC: Raykov's reliability coefficient; AVE: Average variance extracted; SQ: Service quality; NSD: Norwegian Social Science Data Services

\section{References}

1. Gaupta V, Agarwal UA, Khatri N. The relationships between perceived organizational support, affective commitment, psychological contract breach, organizational citizenship behavior and work engagement. J Adv Nurs. 2016;72(11):2806-2817.

2. Slåtten T, Lien G, Svenkerud PJ. The role of organizational attractiveness in an internal marketoriented culture (IMOC): a study of hospital frontline employees. BMC Health Serv Res. 2019;19:307.

3. Currie EJ, Hill RAC. What are the reasons for high turnover in nursing? A discussion of presumed causal factors and remedies. Int J Nur Stu. 2012;49:1180-1189.

4. Kingma M. Nurses on the move: a global overview. Health Serv Res. 2007;42(3):1281-1298.

5. McHugh MD, Kutney-Lee A, Cimiotti JP, Sloane DM, Aiken LH. Nurses' widespread job dissatisfaction, burnout, and frustration with health benefits signal problems for patient care. Health Aff. 2011;30:202-210.

6. Wan Q, Li Z, Zhou W, Shang S. Effects of work environment and job characteristics on the turnover intention of experienced nurses: The mediating role of work engagement, J Adv Nurs. 2018;74:13321341. 
7. Gauci BR, Norman IJ. Factors influencing turnover and absence of nurses: A research review. Int J Nurs Stud. 1997;34:385-394.

8. Kayne B, Jordan ES. Engaging talent. Executive Excellence. 2003;20(8):11.

9. Keyko K, Cummings GG, Yonge 0 , Wong $C A$. Work engagement in professional nursing practice: $A$ systematic review. Int J Nurs Stud. 2016;61:142-164.

10. Bargagliotti LA. Work engagement in nursing: a concept analysis. J Adv Nurs. 2011;68(6):1414-1428.

11. Olsen E, Bjaalid G, Mikkelesen A. Work climate and the mediating role of workplace bullying related to job performance, job satisfaction, and work ability: A study among hospital nurses. J Adv Nurs. 2017;73:2709-2719.

12. Slåtten T, Lien G, Lupina E, Gravingen KA. Promoting an internal market-oriented culture (IMOC) in healthcare services. J Serv Sci Res. 2019;11:157-182.

13. Luthans F, Avolio BJ, Avey JB, Norman SM. Positive psychological capital: measurement and relationship with performance and satisfaction. Pers Psyc. 2007;60:541-572.

14. Schaufeli WB, Salanova M, González-Romá V, Bakker AB. The measurement of engagement and burnout: a two sample confirmatory factor analytic approach. J Happiness Studies. 2002;3:71-92.

15. Schaufeli WB, Bakker AB. Job demands, job resources and their relationship with burnout and engagement, J. Org. Beh. 2004;25:293-315.

16. Tomietto M, Paro E, Sartor R, Maricchio R, Clarizia L, Lucia PD, Pedrinelli G, Finos R. Work engagement and perceived work ability: an evidence-based model to enhance nurses' well-being. $J$ Adv Nurs. 2019;75(9):1933-1942.

17. Kraut Al. Predicting turnover employees from measured job attitudes. Org Beh H Perf. 1975;13:233243.

18. Park JS, Kim TH. Do types of organizational culture matter in nurse job satisfaction and turnover intention? Leadership Health Serv. 2009;22(1):20-38.

19. Moloney W, Boxall P, Parsons M, Cheung G. Factors predicting registered nurse intentions to leave their organization and profession: a job demands-resources framework. J Adv Nurs. 2018;74:864875.

20. Rodwell J, McWilliams J, Gulyas A. The impact of characteristics of nurses' relationship with their supervisor, engagement and trust, on performance behaviors and intent to quit. J Adv Nurs. 2016;73(1):190-200.

21. Stamps PL. Nurses and work satisfaction: an index for measurement, 1997, 2nd edn. Health Adm Press. Chicago, IL.

22. Anaza NA, Rutherford BN. Developing our understanding of patronizing frontline employees. Manag Serv Qual. 2012;22(4):340-358.

23. Alarcon GM, Lyons JB. The relationship of engagement and job satisfaction in working samples. J. Psy. 2011;145:463-480. 
24. Chen SY, Wu WC, Chang CS, Lin CT, Kung JY, Weng HC, Lin YT, Lee SI. Organizational justice, trust, and identification and their effects on organizational commitment in hospital nursing staff. BMC Health Serv Res. 2015;15:363.

25. Dabholkar PA, Shepherd DC, Thorpe DI. A comprehensive framework for service quality: an investigation of critical conceptual and measurement issues through a longitudinal study. J Retailing. 2000;76(2):139-173.

26. Chung BG, Schneider B. Serving multiple masters: role conflict experienced by service employees. J Services Marketing. 2002;16(1):70-87.

27. Slåtten T, Svensson G, Sværi S. Service quality and turnover intention as perceived by employees antecedents and consequences. Personnel Review. 2009;40(2):205-221.

28. Kieft AMM, de Brouwer BJM, Francke AL, Delnoij DMJ. How nurses and their work environment affect patient experiences of the quality of care: a qualitative study. BMC Health Ser Res. 2014;14:410.

29. Abdelhadi N, Drach-Zhavay D. Promoting patient care: work engagement as a mediator between ward service climate and patient-centered care. J Adv Nurs. 2011;68(6):1276-1287.

30. D’Amour D, Goulet L, Labadie JF, Martín-Rodriguez LSM, Pineault R. A model and typology of collaboration between professionals in healthcare organizations. BMC Health Serv Res. 2008;8:188.

31. Kohli AJ, Jaworski BJ. Market orientation: the construct, research propositions, and managerial implications. J Mark. 1990;54(2):90-113.

32. Jaworski BJ, Kohli AK. Market orientation: antecedents and consequences. J Mark. 1993;57(3):5370.

33. Bakker AB, Demerouti E. The job demands-resources model: State of the art. J Manag Psy. 2007;22:309-328.

34. Sullivan Havens D, Warshawsky NE, Vasey J. RN work engagement in generational cohorts: the view from rural US hospitals. J Nurs Manag. 2013;21:927-940.

35. Demirtas O, Akdogan AA. The effect of ethical leadership behavior on ethical climate, turnover intention, and affective commitment. J Bus Ethics. 2015;130:59-67.

36. Banaszak-Holl J, Castle NG, Lin MK, Shrivastwa N, Spreitzer G. The role of organizational culture in retaining nursing workforce. The Gerontologist. 2015;3:462-471.

37. Carlucci D, Schiuma G. Organizational climate as performance driver: health care workers' perception in a large hospital. J Health Manag. 2014;16(4):583-594.

38. Yafang T. Relationship between organizational culture, leadership behavior and job satisfaction. BMC Health Serv Res. 2011;11:98.

39. Hogan SJ, Coote LV. Organizational culture, innovation and performance: a test of Schein's model. J Bus Res. 2014;67(8):1609-1621.

40. Gounaris SP. Internal-market orientation and its measurement. J Bus Res. 2006;59(49):432-448.

41. Bandura A. Social foundation of thought and action. Englewood Cliffs, NJ: Prentice-Hall; 1986. 
42. Trus $\mathrm{M}$, Galdikiene N, Balciunas $\mathrm{S}$, Green $\mathrm{P}$, Helminen $\mathrm{M}$, Suominen T. Connection between organizational culture and climate and empowerment: The perspective of nurse managers. Nurs Health Sci. 2019;21:54-62.

43. Slåtten T. The effect of managerial practice on employee-perceived service quality: the role of emotional satisfaction. Manag Serv Qual. 2009;19(4):431-455.

44. Boshoff C, Allen J. The influence of selected antecedents on frontline staff's perceptions of service recovery performance. Int J Serv Industry Manage. 2000;11(1):63-90.

45. Mehmetoglu M, Jakobsen TG. Applied statistics using Stata - a guide for the social sciences, Sage, London, UK; 2017.

46. Mehmetoglu M. Medsem: a Stata package for statistical mediation analysis. Int J Comput Econ Econometrics. 2018;8(1):63-77.

47. Zhao X, Lynch JG, Chen Q. Reconsidering Baron and Kenny: myths and truths about mediation analysis. J Consumer Res. 2010;37:197-206.

48. Mesfin D, Woldie M, Adamu A, Bekele F. Perceived organizational culture and its relationship with job satisfaction in primary hospitals of Jimma zone and Jimma town administration, correlational study. BMC Health Serv. Res. 2020;20;438.

49. Barrick MR, Thurgood GR, Smith TA, Courtright SH. Collective organizational engagement: linking motivational antecedents, strategic implementation, and firm performance. Acad Manag J. 2015;58(1):111-135.

50. Slåtten T, Lien G. Consequences of employees' collective engagement in knowledge-based service firms. J Serv Science Res. 2016;8:95-129.

51. Cameron KS, Quinn RE. Diagnosing and changing organizational culture: based on the competing value framework. Wiley, New York; 2012.

52. Polston-Murdoch L. An investigation of path-goal theory, relationship of leadership style, supervisorrelated commitment, and gender. Emerging Leadership Journeys. 2013;6(1):13-44.

\section{Figures}




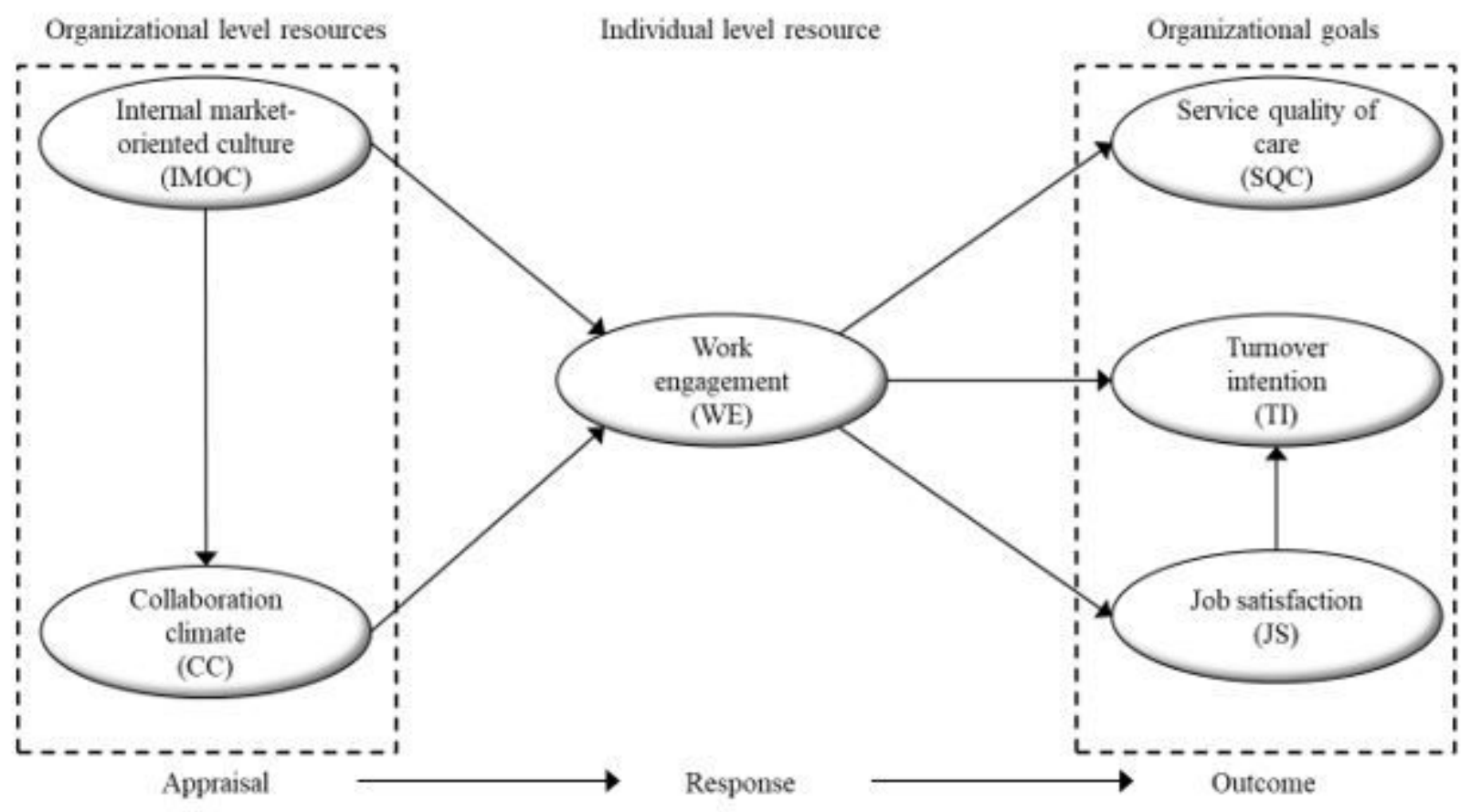

Figure 1

Conceptual model to examine precursors and outcomes of WE

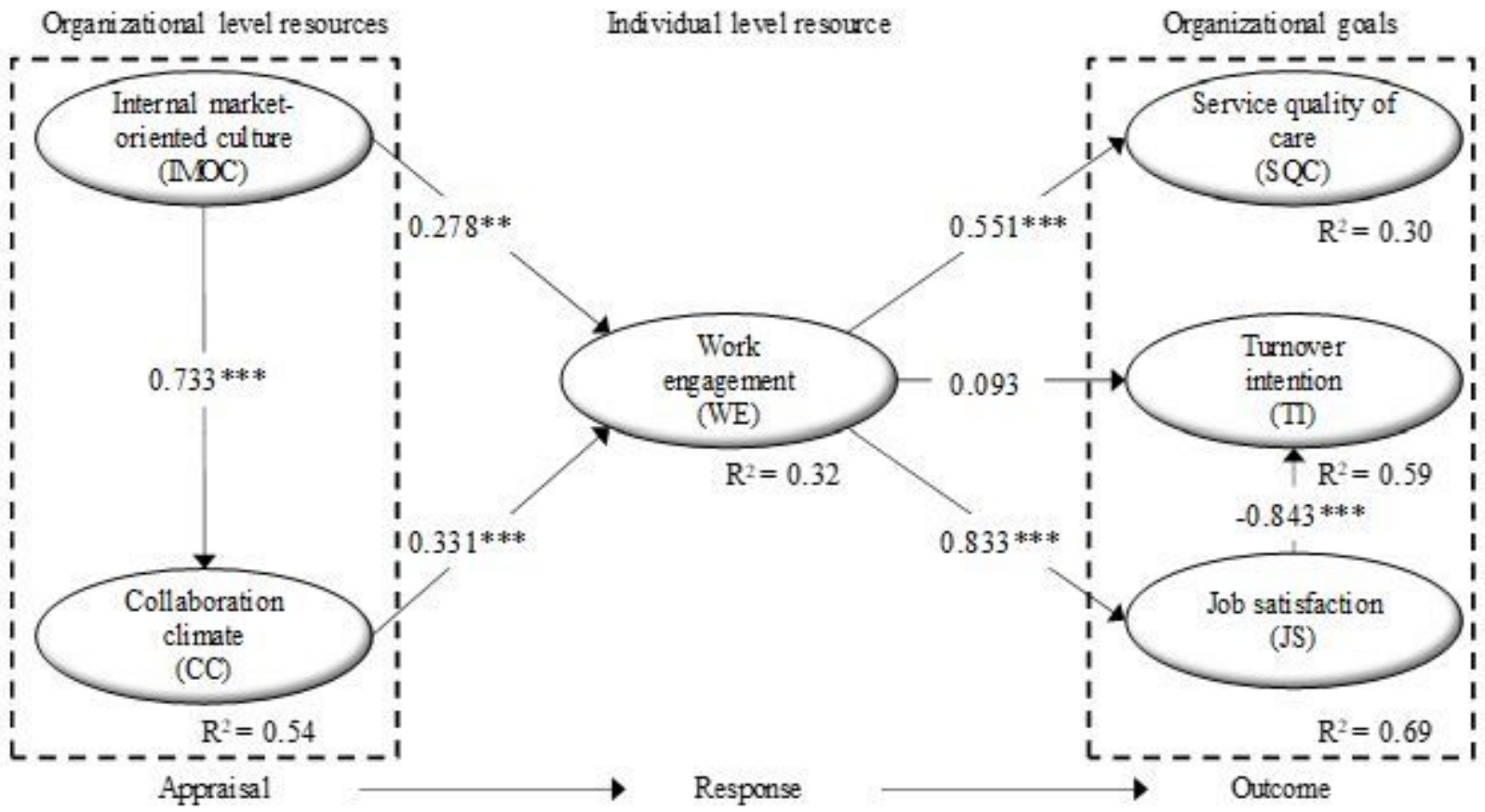

Figure 2

Results of the structural model to analyze the precursors and outcomes of work engagement. Standardized coefficients $(* \star \star p<0.01, * \star p<0.05)$ 